International Journal of Pure and Applied Mathematics

Volume 85 No. 3 2013, 435-455

ISSN: 1311-8080 (printed version); ISSN: 1314-3395 (on-line version)

url: http://www.ijpam.eu

doi: http://dx.doi.org/10.12732/ijpam.v85i3.1

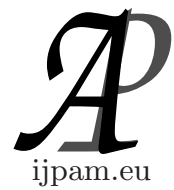

\title{
ON CERTAIN $p$-ADIC BANACH LIMITS OF $p$-ADIC TRIANGULAR MATRIX ALGEBRAS
}

\author{
R.L. Baker \\ University of Iowa \\ Iowa City, Iowa 52242, USA
}

\begin{abstract}
In this paper we investigate the class of $p$-adic triangular UHF (TUHF) Banach algebras. A $p$-adic TUHF Banach algebra is any unital $p$-adic Banach algebra $\mathcal{T}$ of the form $\mathcal{T}=\overline{\cup \mathcal{T}_{n}}$, where $\left(\mathcal{T}_{n}\right)$ is an increasing sequence of $p$-adic Banach subalgebras of $\mathcal{T}$ such that each $\mathcal{T}_{n}$ contains the identity of $\mathcal{T}$ and is isomorphic as an $\Omega_{p}$-algebra to $T_{p_{n}}\left(\Omega_{p}\right)$ for some $p_{n}$, where $T_{p_{n}}\left(\Omega_{p}\right)$ is the algebra of upper triangular $p_{n} \times p_{n}$ matrices over the $p$-adic field $\Omega_{p}$. The main result is that the supernatural number associated to a $p$-adic TUHF Banach algebra is an invariant of the algebra, provided that the algebra satisfies certain local dimensionality conditions.
\end{abstract}

AMS Subject Classification: 12J25, 12J99, 46L99, 46S10

Key Words: $p$-adic Banach algebras, $p$-adic Banach limits, $p$-adic UHF algebras, $p$-adic Triangular UHF algebras

\section{Introduction}

In the article, Triangular UHF algebras [2], the author of the present paper introduced a very concrete class of non-selfadjoint operator algebras which are currently called standard triangular UHF operator algebras. Briefly: a standard triangular UHF (TUHF) operator algebra is any unital Banach algebra that is isometrically isomorphic to a Banach algebra inductive limit of the form $\mathcal{T}=$ $\lim _{\rightarrow}\left(T_{p_{n}} ; \sigma_{p_{n} p_{m}}\right)$. Here $\left(p_{n}\right)$ is a sequence of positive integers such that $p_{m} \mid p_{n}$

Received: December 27, 2011

(c) 2013 Academic Publications, Ltd. url: www.acadpubl.eu 
whenever $m \leq n, T_{p_{n}}$ is the algebra of $p_{n} \times p_{n}$ upper triangular complex matrices and for $m \leq n, \sigma_{p_{n} p_{m}}: T_{p_{m}} \rightarrow T_{p_{n}}$ is the mapping $x \hookrightarrow 1 \otimes x=\operatorname{diag}(x, \ldots, x)$. The main result in [2] is that two standard triangular UHF operator algebras are isometrically isomorphic if, and only if they have the same supernatural number. This result has been extended in at least two different directions. One direction is to view standard triangular UHF operator algebras as special cases of Banach algebra inductive limits of upper triangular matrix algebras, and the and the the goal is to extend the main result in [2] to these inductive limits by using purely Banach-algebraic methods. Such an extension is presented in the paper [2] where it is proved that the supernatural number associated to an arbitrary triangular UHF (TUHF) Banach algebra is an invariant of the algebra, provided that the algebra satisfies certain "local dimensionality conditions." In particular, [2] presents a purely Banach-algebraic formulation of the main result in [2]. A second direction in which the main result in [2] can be extended is to view standard triangular UHF algebras as triangular subalgebras of UHF $C^{*}$-algebras, such that the diagonal of the triangular subalgebra is a canonical masa in the ambient $C^{*}$-algebra. In this vein, the results in [2] have been substantially extended by J.R. Peters, Y.T. Poon and B.H. Wagner [13], and by S.C. Power [11], [12].

The principal results of [3] can be extended in yet a third direction, namely to convert these results into a pure piece of $p$-adic functional analysis (to borrow a turn of phrase used by I Kaplansky [1]). The main result of [3], although relying only on classical complex Banach algebra techniques, makes essential use of the classical spectral theorem (the Riesz functional calculus) for complex Banach algebras. For any prime number $p$, let $\Omega_{p}$ be the $p$-adic counterpart of $\underline{\mathrm{C}}$. Replacing $\underline{\mathrm{C}}$ by $\Omega_{p}$ in the definition of complex TUHF Banach algebras, we obtain the definition of $p$-adic TUHF Banach algebras. The results in [3] for complex TUHF algebras can be duplicated for $p$-adic TUHF algebras, provided that a sufficiently general $p$-adic version of the Riesz functional calculus can be developed for $p$-adic Banach algebras. In the preprint [4] we proved an Extended Spectral Theorem for p-adic Banach Algebras (Theorem 2.10 of [4]). In the present article we use this spectral theorem for $p$-adic Banach algebras to extend the results of [3] to $p$-adic TUHF algebras:- this is the content of Theorem 4.2 of the present paper, which states that the supernatural number associated to an arbitrary $p$-adic TUHF Banach algebra is an isomorphism invariant of the algebra, provided that the algebra satisfies certain "local dimensionality conditions."

In [8] J. Glimm proved that the supernatural number of an arbitrary UHF $C^{*}$-algebra is a complete $C^{*}$-invariant of the algebra. In light of the results on 
$p$-adic TUHF algebras in the present paper, it seems natural to replace $\underline{\mathrm{C}}$ in the definition of complex UHF $C^{*}$-algebras, thereby obtaining the definition of $p$ adic UHF Banach algebras. It then seems natural to ask whether or not the the supernatural number associated to an arbitrary $p$-adic UHF Banach algebra is an isomorphism invariant of the algebra. In the preprint [5] we use the Extended Spectral Theorem for p-adic Banach Algebras obtained in [4] to prove that the supernatural number associated to an arbitrary $p$-adic UHF Banach algebra is an isomorphism invariant of the algebra.

\section{Preliminaries}

In the present section of the paper we put forth the preliminary material on $p$ adic analysis and $p$-adic Banach algebras that is necessary for proving Theorem 4.2 in Section 4.

Let $p$ be a prime number, and let $\mathrm{Q}$ be the field of rational numbers. Let $|\cdot|_{p}$ be the function defined on $\mathrm{Q}$ by

$$
\left|\frac{a}{b}\right|_{p}=p^{\operatorname{ord}_{p} b-\operatorname{ord}_{p} a}, \quad|0|_{p}=0 .
$$

Here $\operatorname{ord}_{p}$ of a non-zero integer is the highest power of $p$ dividing the integer. Then $|\cdot|_{p}$ is a norm on $\mathrm{Q}$. The field $\underline{\mathrm{Q}}_{p}$ is defined to be the completion of $\mathrm{Q}$ under the norm $|\cdot|_{p}$. Unlike the case of the real numbers $\underline{\mathrm{R}}$, whose algebraic closure $\underline{\mathrm{C}}$ is only a quadratic extension of $\underline{\mathrm{R}}$, the algebraic closure $\overline{\mathrm{Q}}_{p}$ of $\underline{\mathrm{Q}}_{p}$ has infinite degree over $\mathrm{Q}_{p}$. However, the norm $|\cdot|_{p}$ on $\underline{Q}_{p}$ can be extended to a norm $|\cdot|_{p}$ on $\overline{\mathrm{Q}}_{p}$. But it turns out that $\underline{\mathrm{Q}}_{p}$ is not complete under this extended norm. Thus, in order to do analysis, we must take a larger field than $\overline{\mathrm{Q}}_{p}$. We denote the completion of $\underline{\mathrm{Q}}_{p}$ under the norm $|\cdot|_{p}$ by $\Omega_{p}$, that is,

$$
\Omega_{p}=\stackrel{\wedge}{\mathrm{Q}}_{p}
$$

where ${ }^{\wedge}$ means completion with respect to $|\cdot|_{p}$. (Note: The symbol " $\underline{\mathrm{C}}_{p}$ " is sometimes used to denote $\Omega_{p}$ (see [9]: page 13). We define $\left|\Omega_{p}\right|_{p}=\left\{|x|_{p}: x \in\right.$ $\left.\Omega_{p}\right\}$.

Definition 2.1. Let $r \geq 0$ be an nonnegative real number, let $a \in \Omega_{p}$ and let $\sigma \subseteq \Omega_{p}$. We have the following definitions.

$$
D_{a}(r)=\left\{x \in \Omega_{p}|\quad| x-\left.a\right|_{p} \leq r\right\} ;
$$




$$
\begin{aligned}
D_{a}\left(r^{-}\right) & =\left\{x \in \Omega_{p}\right. & \mid & \left.|x-a|_{p}<r\right\} ; \\
D_{\sigma}(r) & =\left\{x \in \Omega_{p}\right. & & \operatorname{dist}(x, \sigma) \leq r\} ; \\
D_{\sigma}\left(r^{-}\right) & =\left\{x \in \Omega_{p}\right. & & \operatorname{dist}(x, \sigma)<r\} .
\end{aligned}
$$

If $b \in D_{a}(r)$, then $D_{b}(r)=D_{a}(r)$, and if $b \in D_{a}\left(r^{-}\right)$, then $D_{b}\left(r^{-}\right)=D_{a}\left(r^{-}\right)$. Thus, any point in a disc is its center. Hence $D_{a}(r), D_{a}\left(r^{-}\right)$are both open and closed in the topological sense. It is conventional to take $\inf \emptyset=+\infty$, hence for $r>0$ we have $D_{\emptyset}\left(r^{-}\right)=\emptyset$ and $D_{\emptyset}(r)=\emptyset$ (see [7], page 4).

Lemma 2.2. Let $a \in \Omega_{p}$, and let $0<r \in\left|\Omega_{p}\right|_{p}$. Let $f: D_{a}(r) \rightarrow \Omega_{p}$. Suppose that there exists a sequence $\left(c_{k}\right)$ in $\Omega_{p}$ be such that $\lim _{k \rightarrow \infty} r^{k}\left|c_{k}\right|_{p}=0$, and for all $x \in D_{a}(r)$,

$$
f(x)=\sum_{k=0}^{\infty} c_{k}(x-a)^{k} .
$$

Then $\max _{x \in D_{a}(r)}|f(x)|_{p}$ is attained when $|x-a|_{p}=r$, and we have

$$
\max _{x \in D_{a}(r)}|f(x)|_{p}=\max _{k} r^{k}\left|c_{k}\right|_{p}
$$

Proof. See [9], Lemma 3, page 130.

Definition 2.3. Let $a \in \Omega_{p}$, and let $0<r \in\left|\Omega_{p}\right|_{p}$. Let $\emptyset \neq \sigma \subseteq \Omega_{p}$. A function $f: D_{a}(r) \rightarrow \Omega_{p}$ is said to be Krasner analytic on $D_{a}(r)$ iff $f$ can be represented by a power series on $D_{a}(r)$ of the form $f(x)=\sum_{k=0}^{\infty} c_{k}(x-a)^{k}$, where $\lim _{k \rightarrow \infty} r^{k}\left|c_{k}\right|_{p}=0$. Define $B_{r}(\sigma)$ to be the set of all functions

$$
f: D_{\sigma}(r) \rightarrow \Omega_{p}
$$

such that $f$ is Krasner analytic on $D_{a}(r)$ whenever $a \in \Omega_{p}$ and $D_{a}(r) \subseteq D_{\sigma}(r)$. If $\sigma$ is compact, we define $L(\sigma)=\bigcup\left\{\left.B_{r}(\sigma)|0<r \in| \Omega_{p}\right|_{p}\right\}$, and we call $L(\sigma)$ the set of locally analytic functions on $\sigma$ (see [9], page 136).

Definition 2.4. Let $\emptyset \neq \sigma \subseteq \Omega_{p}$. For $0<r, s \in\left|\Omega_{p}\right|_{p}$ and $a_{1}, \ldots, a_{N}, b_{1}, \ldots$, $b_{M} \in \Omega_{p}$, define the order relation $\left(s, b_{1}, \ldots, b_{M}\right) \leq\left(r, a_{1}, \ldots, a_{N}\right)$ by

$$
\left(s, b_{1}, \ldots, b_{M}\right) \leq\left(r, a_{1}, \ldots, a_{N}\right) \Longleftrightarrow s \leq r \text { and } \bigcup_{i=1}^{M} D_{b_{i}}(s) \subseteq \bigcup_{i=1}^{N} D_{a_{i}}(r) .
$$

For $0<r \in\left|\Omega_{p}\right|_{p}$ and $a_{1}, \ldots, a_{N} \in \Omega_{p}$, define

$$
B_{r, a_{1}, \ldots, a_{N}}=\left\{f: \bigcup_{i=1}^{N} D_{a_{i}}(r) \rightarrow \Omega_{p} \mid f \text { is Krasner analytic on each } D_{a_{i}}(r)\right\} .
$$


Let $I(\sigma)$ to be the set of all $\left(r, a_{1}, \ldots, a_{N}\right)$ such that

(1) $0<r \in\left|\Omega_{p}\right|_{p}$ and $a_{1}, \ldots, a_{N} \in \Omega_{p}$;

(2) $D_{a_{i}}(r) \cap D_{a_{j}}(r)=\emptyset$ for $i \neq j$.

(3) $\sigma \subseteq \bigcup_{i=1}^{N} D_{a_{i}}\left(r^{-}\right)$.

Finally, define

$$
\mathcal{L}(\sigma)=\bigcup\left\{B_{r, a_{1}, \ldots, a_{N}} \mid\left(r, a_{1}, \ldots, a_{N}\right) \in I(\sigma)\right\} .
$$

Lemma 2.5. Let $\emptyset \neq \sigma \subseteq \Omega_{p}$. Then the following statements hold.

(a) The set $I(\sigma)$ is decreasingly filtered under the order relation $\leq$.

(b) Let $\left(r, a_{1}, \ldots, a_{N}\right) \in I(\sigma)$ and $f \in B_{r, a_{1}, \ldots, a_{N}}$. Then $f$ is bounded on $U=\bigcup_{i=1}^{N} D_{a_{i}}(r)$, and we define the uniform norm $\|f\|_{u}$ of $f$ by

$$
\|f\|_{u}=\max _{x \in U}|f(x)|_{p} .
$$

(c) Let $\left(s, b_{1}, \ldots, b_{M}\right),\left(r, a_{1}, \ldots, a_{N}\right) \in I(\sigma)$, with $\left(s, b_{1}, \ldots, b_{N}\right) \leq\left(r, a_{1}, \ldots\right.$, $\left.a_{N}\right)$. Define $V=\bigcup_{i=1}^{M} D_{b_{i}}(s)$, then $\left.f\right|_{V} \in B_{s, b_{1}, \ldots, b_{M}}$ and the mapping $\left.f \mapsto f\right|_{V}$ is continuous on $B_{r, a_{1}, \ldots, a_{N}}$ in the uniform norm.

(d) $\mathcal{L}(\sigma)$ is an algebra over $\Omega_{p}$.

(e) For $\alpha=\left(r, a_{1}, \ldots, a_{N}\right) \in I(\sigma)$, set $B_{\alpha}=B_{r, a_{1}, \ldots, a_{N}}$. For $\beta=\left(s, b_{1}, \ldots\right.$, $\left.b_{M}\right)$ in $I(\sigma)$, with $\beta \leq \alpha$, let $V=\bigcup_{i=1}^{M} D_{b_{i}}(s)$ and define $\varphi_{\beta}^{\alpha}: B_{\alpha} \rightarrow B_{\beta}$ by

$$
\varphi_{\beta}^{\alpha}(f)=\left.f\right|_{V}, \text { all } f \in B_{\alpha} .
$$

Then for $\alpha \geq \beta \geq \gamma \in I(\sigma)$ following conditions are satisfied

$$
\begin{aligned}
\varphi_{\alpha}^{\alpha}(f) & =f, \text { for all } f \in B_{\alpha}, \\
\varphi_{\beta}^{\alpha} \varphi_{\gamma}^{\beta} & =\varphi_{\gamma}^{\alpha} .
\end{aligned}
$$

It is worth noting here that we can define the inverse $\operatorname{limit} \lim B_{\alpha}$ of the system $\left\{B_{\alpha} \mid \alpha \in I(\sigma)\right\}$ and place the projective topology on $\lim _{\longleftarrow} \overleftarrow{B_{\alpha}}$. But we will not make use of this topology in the present paper. 
(f) If $\sigma$ is compact, then $\mathcal{L}(\sigma)=L(\sigma)$, i.e.,

$$
\mathcal{L}(\sigma)=\bigcup\left\{\left.B_{r}(\sigma)|0<r \in| \Omega_{p}\right|_{p}\right\} .
$$

(g) For all $\left(r, a_{1}, \ldots, a_{N}\right) \in I(\sigma)$, if $a_{1}, \ldots, a_{N} \in \sigma$, then $B_{r, a_{1}, \ldots, a_{N}}=B_{r}(\sigma)$.

Proof. See [4], Lemma 1.8.

Definition 2.6. Let $\emptyset \neq \sigma \subseteq \Omega_{p}$ be compact, let $H_{0}(\bar{\sigma})$ denote the set of functions $\varphi: \bar{\sigma} \rightarrow \Omega_{p}$ which are Krasner analytic on the complement $\bar{\sigma}$ of $\sigma$, i.e.,

(1) $\varphi$ is the limit of rational functions whose poles are contained in $\sigma$, the limit being uniform in any set of the form

$$
\bar{D}_{\sigma}(r)=\left\{z \in \Omega_{p} \mid \operatorname{dist}(z, \sigma) \geq r\right\}, \quad \sigma \subseteq D_{\sigma}\left(r^{-}\right), r>0 .
$$

(2) $\lim _{|z|_{p} \rightarrow \infty} \varphi(z)=0$.

The following definition gives the $p$-adic analogue of the classical line integral. Shnirelman introduced this definition in 1938 [16]. The Shnirelman integral can be used to prove $p$-adic analogues of the Cauchy integral theorem, the residue theorem, and the maximum modulus principle of classical complex analysis.

Definition 2.7. (The Shnirelman Integral) Let $r>0$, with $r \in\left|\Omega_{p}\right|_{p}$. Let $a \in \Omega_{p}$, and let $f$ be an $\Omega_{p}$-valued function whose domain contains all $x \in \Omega_{p}$ such that $|x-a|_{p}=r$. Let $\Gamma \in \Omega_{p}$, with $|\Gamma|_{p}=r$. Then the Shnirelman integral of $f$ over the circle

$$
\left\{x \in \Omega_{p}:|x-a|_{p}=r\right\}
$$

is defined to be the following limit, provided the limit exists.

$$
\int_{a, \Gamma} f(x) d x=\lim _{\substack{n \rightarrow \infty \\ p \nmid n}} \frac{1}{n} \sum_{\xi^{n}=1} f(a+\xi \Gamma) .
$$

Theorem 2.8. ( $p$-adic Cauchy Integral Formula) Let $a \in \Omega_{p}$ and $0<r \in$ $\left|\Omega_{p}\right|_{p}$. Let $f$ be Krasner analytic on $D_{a}(r)$, and let $\Gamma \in \Omega_{p}$, with $|\Gamma|_{p}=r$. Then for fixed $z \in \Omega_{p}$, we have, for $m=0,1,2, \ldots$,

$$
\int_{a, \Gamma} \frac{f(x)(x-a)}{(x-z)^{m+1}} d x= \begin{cases}\frac{1}{m !} f^{(m)}(z), & \text { if }|z-a|_{p}<r ; \\ 0, & \text { if }|z-a|_{p}>r .\end{cases}
$$


Proof. See [9], Lemma 4, page 131.

\section{3. $p$-Adic Triangular UHF Algebras}

The following lemma is a key lemma (Lemma 2.1 of [3]) used in proving the main result of [3].

Lemma 3.1. Let $\mathcal{A}$ be a complex Banach algebra. Let $\epsilon>0$ be positive number, and let $I=\left\{e_{i} \mid 1 \leq i \leq n\right\}$ be an orthogonal family of idempotents in $\mathcal{A}$. Then there exists a positive number $\delta(\epsilon, I)>0$ with the following property. Let $\mathcal{B}$ be a unital Banach subalgebra of $\mathcal{A}$, and suppose that $\left\{a_{i} \mid 1 \leq i \leq n\right\}$ is a family of elements in $\mathcal{B}$ such that $\left\|e_{i}-a_{i}\right\| \leq \delta(\epsilon, I)$ for $1 \leq i \leq n$. Then there exists an orthogonal family $\left\{f_{i} \mid 1 \leq i \leq n\right\}$ of idempotents in $\mathcal{B}$ such that $\left\|e_{i}-f_{i}\right\|<\epsilon$ for $1 \leq i \leq n$.

The basic tool used in the proof of Lemma 3.1 is the classical Riesz functional calculus (see [6], prop. 4.5.1, p. 27). In this section we prove Lemma 3.18 , which is the $p$-adic counterpart of Lemma 3.1 for $p$-adic Banach algebras. Theorem 4.2 of the present paper is the exact $p$-adic counterpart of the principal theorem (Theorem 1.1) of [3]. Using the remarks after Definition 3.2 and Theorem 3.15 of this section, and then Lemma 3.18 of this section, the proof of Theorem 1.1 in [3] can be easily adapted to produce a proof of Theorem 4.2. Theorem 3.11 of the present section (Extended Spectral Theorem for p-adic Banach Algebras) is Theorem 2.10 of [4].

Definition 3.2. A p-adic Banach space over $\Omega_{p}$ is a vector space $\mathcal{X}$ over $\Omega_{p}$ together with a norm $\|\cdot\|_{p}$ from $\mathcal{X}$ to the nonnegative real numbers such that for all $x, y \in \mathcal{X}$ : (a) $\|x\|_{p}=0$ if and only if $x=0$; (b) $\|x+y\|_{p} \leq \max \left\{\|x\|_{p},\|y\|_{p}\right\}$; (c) $\|a x\|_{p}=|a|_{p}\|x\|_{p}$; (d) $\mathcal{X}$ is complete under $\|\cdot\|_{p}$. We shall assume that $\|\mathcal{X}\|_{p}=\left|\Omega_{p}\right|_{p}$, i.e., for every $0 \neq x \in \mathcal{X}$ there exists $a \in \Omega_{p}$ such that $\|a x\|_{p}=1$. The dual $\mathcal{X}^{*}$ of a $p$-adic Banach space over $\Omega_{p}$ is defined in the usual way. If $\mathcal{X}$ and $\mathcal{Y}$ are $p$-adic Banach spaces over $\Omega_{p}$, we define $\mathcal{X} \simeq \mathcal{Y}$ to mean that $\mathcal{X}$ and $\mathcal{Y}$ are isometrically isomorphic as $p$-adic Banach spaces over $\Omega_{p}$. A $p$ adic Banach algebra over $\Omega_{p}$ is a $p$-adic Banach space $\mathcal{A}$ over $\Omega_{p}$ such that for $x, y \in \mathcal{A}$, we have $\|x y\|_{p} \leq\|x\|_{p}\|y\|_{p}$. We shall assume that $\mathcal{A}$ has a unit. For $x \in \mathcal{A}$, the spectrum $\sigma_{x}$ of $x$ has the usual meaning, and the resolvent of $x$ is defined by $R(z ; x)=(z-x)^{-1}, z \notin \sigma_{x}$. If $\mathcal{X}$ and $\mathcal{Y}$ are $p$-adic Banach spaces over $\Omega_{p}$, then $B(\mathcal{X}, \mathcal{Y})$ is the vector space of $\Omega_{p}$-linear continuous maps form $\mathcal{X}$ to $\mathcal{Y} . B(\mathcal{X}, \mathcal{Y})$ is a $p$-adic Banach space under the usual operator norm, and 
$B(\mathcal{X})=B(\mathcal{X}, \mathcal{X})$ is a $p$-adic Banach algebra under this operator norm. If $\mathcal{X}$ is a $p$-adic Banach space over $\Omega_{p}$ and $A \in B(\mathcal{X})$ is an operator with compact spectrum $\sigma_{A}$, then $A$ is analytic iff the resolvent $R(z ; A)$ is Krasner analytic in the sense that for all $x \in \mathcal{X}$ and for all $h \in \mathcal{X}^{*}$, function $z \mapsto h(R(z ; A) x)$ is in $H_{0}\left(\bar{\sigma}_{A}\right)$. Let $J$ be any nonempty indexing set, and define $\Omega_{p}(J)$ to be the set of all "sequences" $c=\left(c_{j}\right)_{j \in J}$ in $\Omega_{p}$ such that for every $\epsilon>0$ only finitely many $\left|c_{j}\right|_{p}$ are $>\epsilon$. Define $\|c\|_{p}=\max _{j}\left|c_{j}\right|_{p}$. Then $\Omega_{p}(J)$ is a $p$-adic Banach space over $\Omega_{p}$ (see [14], Corollary 1, page 185). The notation $\Omega_{p}(J)$ is used in [9], page 143. However the usual notation for $\Omega_{p}(J)$ is $c_{0}\left(J ; \Omega_{p}\right)$, which is used in [14], page 185. Let $\mathcal{A}$ be a $p$-adic Banach algebra and let $\mathcal{X}$ be a $p$-adic Banach space. Let $A \in \mathcal{A}$ have compact spectrum $\sigma_{A} \neq \emptyset$. Then we say that $A$ is $\mathcal{X}$-analytic iff there exists an $\Omega_{p}$-monomorphism $\theta: \mathcal{A} \rightarrow B(\mathcal{X})$ such that $\theta(\mathcal{A})$ is a $p$-adic Banach subalgebra of $B(\mathcal{X}), \theta^{-1}: \theta(\mathcal{A}) \rightarrow \mathcal{A}$ is bounded and $\theta(A)$ is analytic in $B(\mathcal{X})$. Such a monomorphism $\theta: \mathcal{A} \rightarrow B(\mathcal{X})$ is said to be an embedding of $\mathcal{A}$ into $B(\mathcal{X})$. Let $B \in \mathcal{A}$. Then $B$ is $\mathcal{A}$-analytic iff the following two conditions hold:

(a) For every $r>0,\|R(x ; B)\|_{p}$ is bounded on the complement $\bar{D}_{\sigma_{B}}(r)$ of $D_{\sigma_{B}}\left(r^{-}\right)$.

(b) There exists a sequence $\left(B_{k}\right)$ in $\mathcal{A}$ and a sequence $\left(\mathcal{X}_{k}\right)$ of $p$-adic Banach spaces $\mathcal{X}_{k}$ of the form $\mathcal{X}_{k} \simeq \Omega_{p}\left(J_{k}\right)$, with $J_{k} \neq \emptyset$, such that $\sigma_{B_{k}} \neq \emptyset$ is compact, $B_{k}$ is $\mathcal{X}_{k}$-analytic and $\lim _{k \rightarrow \infty}\left\|B-B_{k}\right\|_{p}=0$.

Remark. Let $\mathcal{X} \simeq \Omega_{p}(J)$, where $J \neq \emptyset$. Let $\mathcal{A}$ be a $p$-adic Banach algebra over $\Omega_{p}$. If $A \in \mathcal{A}$ has nonempty compact spectrum $\sigma_{A}$, and if $A$ is $\mathcal{X}$-analytic, then $A$ is $\mathcal{A}$-analytic (see Lemma 2.13 of [4]). If $J$ is nonempty and finite, and there exists an embedding of $\mathcal{A}$ into $B(\mathcal{X})$, then every element of $\mathcal{A}$ is $\mathcal{X}$-analytic, hence every element of $\mathcal{A}$ is $\mathcal{A}$-analytic (see Lemma 3.2 of [4]).

Definition 3.3. Let $\mathcal{A}$ be a $p$-adic Banach algebra over $\Omega_{p}$. Let $A \in \mathcal{A}$ have spectrum $\sigma_{A} \neq \emptyset$. Define $\mathcal{F}(A)=\mathcal{L}\left(\sigma_{A}\right)$.

Definition 3.4. (Operator-Valued Shnirelman Integral) Let $\mathcal{A}$ be a $p$-adic Banach algebra over $\Omega_{p}$. Let $a \in \Omega_{p}$, and let $0<r \in\left|\Omega_{p}\right|_{p}$. Let $\Gamma \in \Omega_{p}$, with $|\Gamma|_{p}=r$. Let $F$ be a function defined on the circle $\left\{z \in \Omega_{p}:|z-a|_{p}=r\right\}$ into $\mathcal{A}$. Then we define

$$
\int_{a, \Gamma} F(x) d x=\lim _{\substack{n \rightarrow \infty \\ p \nmid n}} \frac{1}{n} \sum_{\xi^{n}=1} F(a+\xi \Gamma),
$$

provided that this limit exists. 
Lemma 3.5. Let $\mathcal{A}$ be a $p$-adic Banach algebra. Let $A \in \mathcal{A}$ be $\mathcal{A}$-analytic. Let $\left(A_{k}\right)$ be a sequence in $\mathcal{A}$ and $\left(\mathcal{X}_{k}\right)$ a sequence of $p$-adic Banach spaces $\mathcal{X}_{k}$ of the form $\mathcal{X}_{k} \simeq \Omega_{p}\left(J_{k}\right)$, with $J_{k} \neq \emptyset$, such that $\sigma_{A_{k}} \neq \emptyset$ is compact, $A_{k}$ is $\mathcal{X}_{k}$-analytic and $\lim _{k \rightarrow \infty}\left\|A-A_{k}\right\|_{p}=0$. Let $A$ have spectrum $\sigma_{A}$. Let $0<r_{2} \leq r_{1}$ be in $\left|\Omega_{p}\right|_{p}$, and let $\Gamma_{1}, \Gamma_{2}$ be in $\Omega_{p}$, with $\left|\Gamma_{1}\right|_{p}=r_{1},\left|\Gamma_{2}\right|_{p}=r_{2}$. Assume that $a_{1}, \ldots, a_{M} ; b_{1}, \ldots, b_{N} \in \Omega_{p}$ are given, with

$$
\sigma_{A} \subseteq \bigcup_{i=1}^{M} D_{a_{i}}\left(r_{1}^{-}\right) \text {and } \sigma_{A} \subseteq \bigcup_{i=1}^{N} D_{b_{i}}\left(r_{2}^{-}\right)
$$

where the $D_{a_{i}}\left(r_{1}\right)$ are disjoint and the $D_{b_{i}}\left(r_{2}\right)$ are disjoint. Let $f$ be Krasner analytic on the $D_{a_{i}}\left(r_{1}\right)$ and on the $D_{b_{i}}\left(r_{2}\right)$. Then the following limits exist and are equal:

$\lim _{k \rightarrow \infty} \sum_{i=1}^{M} \int_{a_{i}, \Gamma_{1}} f(x)\left(x-a_{i}\right) R\left(x ; A_{k}\right) d x=\lim _{k \rightarrow \infty} \sum_{i=1}^{N} \int_{b_{i}, \Gamma_{2}} f(x)\left(x-b_{i}\right) R\left(x ; A_{k}\right) d x$.

Proof. See [4], Lemma 2.5.

Lemma 3.6. Let $\mathcal{A}$ be a $p$-adic Banach algebra. Let $A \in \mathcal{A}$ be $\mathcal{A}$-analytic with spectrum $\sigma_{A}$. Let $\left(A_{k}\right)$ be a sequence in $\mathcal{A}$ and $\left(\mathcal{X}_{k}\right)$ a sequence of $p$-adic Banach spaces $\mathcal{X}_{k}$ of the form $\mathcal{X}_{k} \simeq \Omega_{p}\left(J_{k}\right)$, with $J_{k} \neq \emptyset$, such that $\sigma_{A_{k}} \neq \emptyset$ is compact, $A_{k}$ is $\mathcal{X}_{k}$-analytic and $\lim _{k \rightarrow \infty}\left\|A-A_{k}\right\|_{p}=0$. Let $0<r \in\left|\Omega_{p}\right|_{p}$, and let $\Gamma \in \Omega_{p}$, with $|\Gamma|_{p}=r$. Assume that $a_{1}, \ldots, a_{N} \in \Omega_{p}$ are given, with

$$
\sigma_{A} \subseteq \bigcup_{i=1}^{N} D_{a_{i}}\left(r^{-}\right)
$$

where the $D_{a_{i}}(r)$ are disjoint. Assume that $f$ is Krasner analytic on the $D_{a_{i}}(r)$. Then the following limits exist and are equal.

$\lim _{\substack{n \rightarrow \infty \\ p \nmid n}} \sum_{i=1}^{N} \frac{1}{n} \sum_{\xi^{n}=1} f\left(x_{\xi}^{i}\right)\left(x_{\xi}^{i}-a_{i}\right) R\left(x_{\xi}^{i} ; A\right)=\lim _{k \rightarrow \infty} \sum_{i=1}^{N} \int_{a_{i}, \Gamma} f(x)\left(x-a_{i}\right) R\left(x ; A_{k}\right) d x$,

where for each $1 \leq i \leq N$ and $\xi \in \Omega_{p}$, we set $x_{\xi}^{i}=a_{i}+\xi \Gamma$. Therefore the sum

$$
\sum_{i=1}^{N} \int_{a_{i}, \Gamma} f(x)\left(x-a_{i}\right) R(x ; A) d x
$$


exits and we have

$$
\sum_{i=1}^{N} \int_{a_{i}, \Gamma} f(x)\left(x-a_{i}\right) R(x ; A) d x=\lim _{k \rightarrow \infty} \sum_{i=1}^{N} \int_{a_{i}, \Gamma} f(x)\left(x-a_{i}\right) R\left(x ; A_{k}\right) d x .
$$

Proof. See [4], Lemma 2.6.

Definition 3.7. Let $\mathcal{A}$ be a $p$-adic Banach algebra. Let $A \in \mathcal{A}$ be $\mathcal{A}$ analytic with spectrum $\sigma_{A}$. Let $\left(A_{k}\right)$ be a sequence in $\mathcal{A}$ and $\left(\mathcal{X}_{k}\right)$ a sequence of $p$-adic Banach spaces $\mathcal{X}_{k}$ of the form $\mathcal{X}_{k} \simeq \Omega_{p}\left(J_{k}\right)$, with $J_{k} \neq \emptyset$, such that $\sigma_{A_{k}} \neq \emptyset$ is compact, $A_{k}$ is $\mathcal{X}_{k}$-analytic and $\lim _{k \rightarrow \infty}\left\|A-A_{k}\right\|_{p}=0$. Let $f \in \mathcal{F}(A)$. Let $0<r \in\left|\Omega_{p}\right|_{p}$, and let $\Gamma \in \Omega_{p}$, with $|\Gamma|_{p}=r$. Assume that $a_{1}, \ldots, a_{N} \in \Omega_{p}$ are given, with

$$
\sigma_{A} \subseteq \bigcup_{i=1}^{N} D_{a_{i}}\left(r^{-}\right)
$$

where the $D_{a_{i}}(r)$ are disjoint. Assume that $f$ is Krasner analytic on the $D_{a_{i}}(r)$. By Lemma 3.6, (1) implies that

$$
\sum_{i=1}^{N} \int_{a_{i}, \Gamma} f(x)\left(x-a_{i}\right) R(x ; A) d x=\lim _{k \rightarrow \infty} \sum_{i=1}^{N} \int_{a_{i}, \Gamma} f(x)\left(x-a_{i}\right) R\left(x ; A_{k}\right) d x .
$$

Lemma 3.5 implies that

$$
\lim _{k \rightarrow \infty} \sum_{i=1}^{N} \int_{a_{i}, \Gamma} f(x)\left(x-a_{i}\right) R\left(x ; A_{k}\right) d x
$$

does not depend on $a_{1}, \ldots, a_{N}, r, \Gamma$, hence we may define $f(A)$ by

$$
f(A)=\sum_{i=1}^{N} \int_{a_{i}, \Gamma} f(x)\left(x-a_{i}\right) R(x ; A) d x .
$$

Definition 3.8. Let $K$ be an arbitrary field. Let $\mathcal{A}$ be a unital algebra over $K$. A family of elements $\left\{e_{i j} \mid 1 \leq i \leq j \leq n\right\}$ in $\mathcal{A}$ is said to be a triangular system of matrix units in $\mathcal{A}$ if for $1 \leq i \leq j \leq n$ the following conditions are satisfied.

(i) $e_{i j} \neq 0$ 
(ii) $\left\{e_{i i} \mid 1 \leq i \leq n\right\}$ is an orthogonal family of idempotents such that $\sum_{i=1}^{n} e_{i i}=1$;

(iii) for $1 \leq i \leq p \leq n$ and $1 \leq q \leq j \leq n$, we have

$$
e_{i p} e_{q j}= \begin{cases}e_{i j}, & \text { if } \mathrm{p}=\mathrm{q} ; \\ 0, & \text { otherwise } .\end{cases}
$$

A family of elements $\left\{f_{i j} \mid 1 \leq i, j \leq n\right\}$ in $\mathcal{A}$ is said to be a system of matrix units in $\mathcal{A}$ if

(iv) $f_{i j} \neq 0$

(v) $\sum_{i=1}^{n} f_{i i}=1$;

(vi) for $1 \leq i, j, p, q \leq n$, we have $f_{i p} f_{q j}=\delta_{p q} f_{i j}$.

Definition 3.9. A $p$-adic Banach algebra $\mathcal{T}$ over $\Omega_{p}$ is said to be a $p$ adic triangular (TUHF) Banach algebra if there exists an increasing sequence $\left(\mathcal{T}_{n}\right)$ of finite-dimensional $p$-adic Banach subalgebras of $\mathcal{T}$ such that each $\mathcal{T}_{n}$ is generated as a $p$-adic Banach algebra over $\Omega_{p}$ by a triangular system $\left\{e_{i j}^{(n)}\right.$ | $\left.1 \leq i \leq j \leq p_{n}\right\}$ of matrix units in $\mathcal{T}$, and $\mathcal{T}=\overline{\cup \mathcal{T}_{n}}$.

Definition 3.10. Let $\left(p_{n}\right)$ be a sequence of positive integers. The supernatural number of $\left(p_{n}\right)$ is the function $N\left[\left(p_{n}\right)\right]$, defined on the set of prime numbers, such that for any prime number $q$,

$$
N\left[\left(p_{n}\right)\right](q)=\sup \left\{m\left|\exists n: q^{m}\right| p_{n}\right\} .
$$

Theorem 3.11. (Extended Spectral Theorem for $p$-adic Banach Algebras) Let $\mathcal{A}$ be a $p$-adic Banach algebra over $\Omega_{p}$. Let $A \in \mathcal{A}$ be $\mathcal{A}$-analytic, with spectrum $\sigma_{A}$. Let $f, g \in \mathcal{F}\left(\sigma_{A}\right)$. Let $0<r \in\left|\Omega_{p}\right|_{p}$, and let $\Gamma$ be in $\Omega_{p}$, with $|\Gamma|_{p}=r$. Assume that $a_{1}, \ldots, a_{N}$ in $\Omega_{p}$ are given, with

$$
\sigma_{A} \subseteq \bigcup_{i=1}^{N} D_{a_{i}}\left(r^{-}\right)
$$

where the $D_{a_{i}}(r)$ are disjoint. Finally, suppose that $f$ and $g$ are Krasner analytic on each $D_{a_{i}}(r)$. By (1) and Definition 3.7, $f(A)$ and $g(A)$ are well-defined, with

$$
f(A)=\sum_{i=1}^{N} \int_{a_{i}, \Gamma} f(x)\left(x-a_{i}\right) R(x ; A) d x,
$$




$$
g(A)=\sum_{i=1}^{N} \int_{a_{i}, \Gamma} g(x)\left(x-a_{i}\right) R(x ; A) d x .
$$

Let $g \in \mathcal{F}(A)$, and let $\alpha, \beta \in \Omega_{p}$. Then $\alpha f+\beta g, f \cdot g \in \mathcal{F}(A)$, and

(2) $(\alpha f+\beta g)(A)=\alpha f(A)+\beta g(A)$;

(3) $(f \cdot g)(A)=f(A) g(A)$;

(4) The mapping $h \mapsto h(A)$ is continuous on $B_{r, a_{1}, \ldots, a_{N}}$.

Proof. See [4], Theorem 2.10.

Theorem 3.12. Let $\mathcal{A}$ be a $p$-adic $p$-adic Banach algebra. Let $A \in \mathcal{A}$ have spectrum $\sigma_{A}$, and assume that $A$ is $\mathcal{A}$-analytic. Let $f \in \mathcal{F}(A)$. Let $0<r$ be in $\left|\Omega_{p}\right|_{p}$, and let $\Gamma$ be in $\Omega_{p}$, with $|\Gamma|_{p}=r$. Assume that $a_{1}, \ldots, a_{N}$ in $\Omega_{p}$ are given, with

$$
\sigma_{A} \subseteq \bigcup_{i=1}^{N} D_{a_{i}}\left(r^{-}\right),
$$

where the $D_{a_{i}}(r)$ are disjoint and $f$ is Krasner analytic on the $D_{a_{i}}(r)$. Then for any $\epsilon>0$ there exists a $\delta>0$ such that if $B \in \mathcal{A}$ is $\mathcal{A}$-analytic, with $\|A-B\|_{p}<\delta$, then

$$
\sigma_{B} \subseteq \bigcup_{i=1}^{N} D_{a_{i}}(r),
$$

$f \in \mathcal{F}(B)$ and $\|f(A)-f(B)\|_{p}<\epsilon$.

Proof. See [4], Theorem 2.8 .

Lemma 3.13. Let $K$ be a field and let $e \in K$ be an idempotent. If $0,1 \neq z \in K$, then

$$
(z-e)^{-1}=\frac{(z-1)+e}{z(z-1)} .
$$

Proof. A calculation shows that $(z-e)[(z-1)+e]=z(z-1) \neq 0$.

Lemma 3.14. Let $K$ be a nonarchimedean field, i.e., for all $x, y \in K$, $|x+y| \leq \max \{|x|,|y|\}$. Let $0<r<1$. Then

(a) $D_{0}(r) \cap D_{1}(r)=\emptyset$.

(b) If $z \in K$ with $|z(z-1)|<r$, then $z \in D_{0}\left(r^{-}\right) \cup D_{1}\left(r^{-}\right)$.

Proof. Because $K$ is nonarchimedean, if $\lambda, \mu \in K$, with $|\lambda|>|\mu|$, then $|\lambda+\mu|=\lambda$ (see [10], Theorem, p. 5). To prove (a), suppose that $\lambda \in D_{0}(r) \cap$ $D_{1}(r)$. Then $|\lambda| \leq r<1$, hence $|\lambda-1|=1$, which contradicts $\lambda \in D_{1}(r)$, i.e., 
$|\lambda-1| \leq r<1$. This proves (a). To prove (b), suppose that $|\lambda(\lambda-1)|<r$, i.e., $|\lambda||\lambda-1|<r$. There are three cases to consider. In the first Case, $|\lambda|>1$. Then $|\lambda-1|=|\lambda|$, and hence $r>|\lambda||\lambda-1|=|\lambda|^{2}$, which gives $r>|\lambda|^{2}$, i.e., $1>\sqrt{r}>|\lambda|$. This contradiction shows that the first case can not hold. In the second case, $|\lambda|=1$. Then $r>|\lambda||\lambda-1|=|\lambda-1|$, and hence $\lambda \in D_{1}\left(r^{-}\right)$. Finally, in the third case, $|\lambda|<1$. Then we have $|\lambda-1|=1$, and hence we get $r>|\lambda||\lambda-1|=|\lambda|$, consequently, $\lambda \in D_{0}\left(r^{-}\right)$. This proves (b).

Lemma 3.15. Let $\mathcal{A}$ be a $p$-adic Banach algebra over $\Omega_{p}$. Let $E \in \mathcal{A}$ be an idempotent. Then for all $r>0,\|R(x ; E)\|_{p}$ is bounded on $\bar{D}_{\sigma_{E}}(r)$.

Proof. Let $r>0$ be arbitrary. Suppose that $E=0$, then $\sigma_{E}=\{0\}$. If $x \in \bar{D}_{\sigma_{E}}(r)$, then $|x|_{p} \geq r$, and hence $\|R(x ; E)\|_{p}=|x|_{p}^{-1} \leq \frac{1}{r}$. This shows that $\|R(x ; E)\|_{p}$ is bounded on $\bar{D}_{\sigma_{E}}(r)$. Now suppose that $E=1$, then $\sigma_{E}=\{1\}$. If $x \in \bar{D}_{\sigma_{E}}(r)$, then $|x-1|_{p} \geq r$, and hence $\|R(x ; E)\|_{p}=|x-1|_{p}^{-1} \leq \frac{1}{r}$. We conclude that $\|R(x ; E)\|_{p}$ is bounded on $\bar{D}_{\sigma_{E}}(r)$. Now assume that $E \neq$ 0,1 , then $\sigma_{E}=\{0,1\}$. Let $x \in \Omega_{p}$, with $|x|_{p}>\|E\|_{p}$. Then by Theorem 2.1(a) of [4], $\left(1-\frac{E}{x}\right)^{-1}$ exists and $\left(1-\frac{E}{x}\right)^{-1}=\sum_{n=0}^{\infty}\left(\frac{E}{x}\right)^{n}$. Hence $(x-E)^{-1}=x^{-1}\left(1-\frac{E}{x}\right)^{-1}$ exists and

$$
\begin{aligned}
\left\|(x-E)^{-1}\right\|_{p} & =|x|_{p}^{-1}\left\|\left(1-\frac{E}{x}\right)^{-1}\right\|_{p}=|x|_{p}^{-1}\left\|\sum_{n=0}^{\infty}\left(\frac{E}{x}\right)^{n}\right\|_{p} \\
& \leq|x|_{p}^{-1} \sum_{n=0}^{\infty}\left(\frac{\|E\|_{p}}{|x|_{p}}\right)^{n} \\
& =|x|_{p}^{-1}\left[1-\frac{\|E\|_{p}}{|x|_{p}}\right]^{-1} .
\end{aligned}
$$

It follows that $\lim _{|x|_{p} \rightarrow \infty}\|R(x ; E)\|_{p}=0$. Let $M \geq 1, r^{2},\|E\|_{p}$ be so large that

$$
\|R(x ; E)\|_{p} \leq 1 \text { for }|x|_{p} \geq M .
$$

Let $x \in \bar{D}_{\sigma_{E}}(r)$, with $|x|_{p} \leq M$. Then $|x|_{p}=|x-0|_{p} \geq r$ and $|x-1|_{p} \geq r$, hence we have

$$
\frac{1}{|x|_{p}} \cdot \frac{1}{|x-1|_{p}} \leq \frac{1}{r^{2}}
$$


Because $E \neq 0,1$, Lemma 3.13 implies that for $x \neq 0,1$,

$$
R(x ; E)=(x-E)^{-1}=\frac{(x-1)+E}{x(x-1)} .
$$

Therefore we get

$$
\begin{aligned}
\|R(x ; E)\|_{p} & =\frac{\|(x-1)+E\|_{p}}{|x|_{p}|x-1|_{p}} \\
& \leq \frac{\max \left\{|x-1|_{p},\|E\|_{p}\right\}}{r^{2}} \\
& \leq \frac{\max \left\{1,|x|_{p},\|E\|_{p}\right\}}{r^{2}} \\
& \leq \frac{\max \left\{1, M,\|E\|_{p}\right\}}{r^{2}} \\
& =\frac{M}{r^{2}} .
\end{aligned}
$$

Thus, if we define $N_{r}=\frac{M}{r^{2}}$, we see that

$$
\|R(x ; E)\|_{p} \leq N_{r} \text { for all } x \in \bar{D}_{\sigma_{E}}(r) .
$$

Hence for all $r>0,\|R(x ; E)\|_{p}$ is bounded on $\bar{D}_{\sigma_{E}}(r)$.

Remark. Let $\mathcal{T}$ be a $p$-adic TUHF algebra over $\Omega_{p}$. Let $A \in \mathcal{T}$ have spectrum $\sigma_{A}$. Assume that for all $r>0,\|R(x ; A)\|_{p}$ is bounded on the complement $\bar{D}_{\sigma_{A}}(r)$ of $D_{\sigma_{A}}\left(r^{-}\right)$. According to Theorem 3.3 of [4], $A$ is then $\mathcal{T}$-analytic. Hence by Theorem 3.15, every idempotent $E \in \mathcal{T}$ is $\mathcal{T}$-analytic.

Theorem 3.16. Let $\mathcal{A}$ be a $p$-adic Banach algebra over $\Omega_{p}$. Let $0,1 \neq e \in$ $\mathcal{A}$ be an idempotent such that $e$ is $\mathcal{A}$-analytic. Let $r \in\left|\Omega_{p}\right|_{p}$ with $0<r<1$, and define

$$
f(x)= \begin{cases}0, & \text { if } x \in D_{0}(r) \\ 1, & \text { if } x \in D_{1}(r) .\end{cases}
$$

Then $f \in \mathcal{F}(e)$ and $f(e)=e$.

Proof. Since $0,1 \neq e$ and $e$ is an idempotent, we have $\sigma_{e}=\{0,1\}$. Because $0<r<1$, Lemma 3.14 implies that

$$
\sigma_{e} \subseteq D_{0}\left(r^{-}\right) \cup D_{1}\left(r^{-}\right), \quad D_{0}(r) \cap D_{1}(r)=\emptyset
$$


Now, $f$ is Krasner analytic on $D_{0}\left(r^{-}\right)$and on $D_{1}\left(r^{-}\right)$, and hence $f \in \mathcal{F}(e)$. Let $\Gamma \in \Omega_{p}$, with $|\Gamma|_{p}=r$. By Theorem 3.11 and Lemma 3.13, (1) implies that

$$
\begin{aligned}
f(e) & =\int_{0, \Gamma} f(x)(e-a)^{-1} d x+\int_{1, \Gamma} f(x)(e-a)^{-1} d x \\
& =\int_{0, \Gamma}(0)(e-a)^{-1} d x+\int_{1, \Gamma}(1)(e-a)^{-1} d x \\
& =\int_{1, \Gamma}\left[\frac{(x-1)+e}{x(x-1)}\right] d x=\int_{1, \Gamma}\left(\frac{x-1}{x}\right) \frac{d x}{x-1}+\left[\int_{1, \Gamma} \frac{d x}{x(x-1)}\right] e .
\end{aligned}
$$

By Theorem 2.8 we have

$$
\int_{1, \Gamma}\left(\frac{x-1}{x}\right) \frac{d x}{x-1}=\left.\left(\frac{x-1}{x}\right)\right|_{x=1}=0
$$

and

$$
\int_{1, \Gamma} \frac{d x}{x(x-1)}=\int_{1, \Gamma}\left(\frac{x-1}{x}\right) \frac{d x}{(x-1)^{2}}=\left.\frac{d}{d x}\left(\frac{x-1}{x}\right)\right|_{x=1}=1 .
$$

Therefore we get that $f(e)=(0)+(1) e=e$. This completes the proof of the theorem.

Theorem 3.17. Let $\mathcal{A}$ be a $p$-adic Banach algebra over $\Omega_{p}$. Let $e \in \mathcal{A}$ be an idempotent such that $e$ is $\mathcal{A}$-analytic. Let $\epsilon>0$ be arbitrary. Then there exists a positive number $\gamma(\epsilon, e)>0$ with the following property. Let $\mathcal{B}$ be a unital p-adic Banach subalgebra of $\mathcal{A}$, and let $a \in \mathcal{B}$ be $\mathcal{A}$-analytic such that $\|e-a\|_{p}<\gamma(\epsilon, e)$. Then there exists an idempotent $f \in \mathcal{B}$ such that

$$
\|e-f\|<\epsilon .
$$

Proof. We may assume that $e \neq 0,1$. Let $0<r<1$ be arbitrary. By Lemma 3.14, $D_{0}(r) \cap D_{1}(r)=\emptyset$. Define

$$
g(x)= \begin{cases}0, & \text { if } x \in D_{0}(r) \\ 1, & \text { if } x \in D_{1}(r)\end{cases}
$$

Then by Theorem 3.16, $g \in \mathcal{F}(e)$ and $g(e)=e$. For $z \in \Omega_{p}$ define $p(z)=$ $z(z-1)$. By the $p$-adic spectral mapping theorem ( [10], p. 111) for all $a \in \mathcal{A}$ 
we have $\sigma_{p(a)}=\left\{z(z-1) \mid z \in \sigma_{a}\right\}$. Because $e^{2}=e$, there exists a $\delta_{1}>0$ such that if $a \in \mathcal{A}$ with $\|a-e\|_{p}<\delta_{1}$, then $\left\|a^{2}-a\right\|_{p}<r$. Then for $\|a-e\|_{p}<\delta_{1}$, we have (see [10], Theorem 6, p. 114 and Theorem 2, p. 107)

$$
\max _{z \in \sigma_{p(a)}}|z|_{p}=r_{\sigma_{p(a)}} \leq \nu(p(a)) \leq\|p(a)\|_{p}<r
$$

and hence for $w \in \sigma_{a}$,

$$
|w|_{p}|w-1|_{p} \leq \max _{z \in \sigma_{a}}|z(z-1)|=\max _{z \in \sigma_{p(a)}}|z|_{p}<r .
$$

Therefore, by Lemma 3.14, if $\|a-e\|_{p}<\delta_{1}$, then

$$
\sigma_{a} \subseteq D_{0}\left(r^{-}\right) \cup D_{1}\left(r^{-}\right) .
$$

By Theorem 3.12 there exists a $\delta_{2}>0$ such that if $a \in \mathcal{A}$ is $\mathcal{A}$-analytic and $\|a-e\|_{p}<\delta_{2}$, then $g \in \mathcal{F}(a)$ and $\|g(e)-g(a)\|_{p}<\epsilon$, i.e., $\|e-g(a)\|_{p}<\epsilon$. Define

$$
\gamma(\epsilon, e)=\min \left\{\delta_{1}, \delta_{2},\right\}
$$

Let $a \in \mathcal{B}$ be $\mathcal{A}$-analytic, with $\|a-e\|_{p}<\gamma(\epsilon, e)$. Because $a$ is $\mathcal{A}$-analytic, $a$ satisfies the hypothesis of Theorem 3.11 and hence we may define $f=g(a)$. Then by Theorem 3.11 we get $f^{2}=g^{2}(a)=g(a)=f$ and $\|e-f\|_{p}<\epsilon$. Because $f \in \mathcal{B}$, this completes the proof of the theorem.

Lemma 3.18. Let $\mathcal{A}$ be a $p$-adic Banach algebra over $\Omega_{p}$. Let $\epsilon>0$ be positive number, and let $I=\left\{e_{i} \mid 1 \leq i \leq n\right\}$ be an orthogonal family of $\mathcal{A}$-analytic idempotents in $\mathcal{A}$. Then there exists a positive number $\delta(\epsilon, I)>0$ with the following property. Let $\mathcal{B}$ be a unital p-adic Banach subalgebra of $\mathcal{A}$ such that each member of $\mathcal{B}$ is $\mathcal{A}$-analytic, and suppose that $\left\{a_{i} \mid 1 \leq i \leq n\right\}$ is a family of elements in $\mathcal{B}$ such that $\left\|e_{i}-a_{i}\right\| \leq \delta(\epsilon, I)$ for $1 \leq i \leq n$. Then there exists an orthogonal family $\left\{f_{i} \mid 1 \leq i \leq n\right\}$ of idempotents in $\mathcal{B}$ such that $\left\|e_{i}-f_{i}\right\|<\epsilon$ for $1 \leq i \leq n$.

Proof. The proof that we give of this lemma is modeled on the proof of Lemma 1.7 of [8]. We prove the lemma by induction on $n$. Each member of $\mathcal{B}$ is $\mathcal{A}$-analytic, hence the case $n=1$ follows from Lemma 3.17. Assume that the lemma is true for $n \geq 1$, and let $\epsilon>0$ be a given positive number. Let $J=\left\{e_{i} \mid 1 \leq i \leq n+1\right\}$ be an orthogonal family of $\mathcal{A}$-analytic idempotents in $\mathcal{A}$. Without loss, we may assume that $\epsilon<1$. Define $\mu=\max \left\{\left\|e_{i}\right\|: 1 \leq\right.$ $i \leq n+1\}+1$. Let $\gamma\left(\epsilon, e_{n+1}\right)=\gamma>0$ be the positive number that Theorem 3.17 assigns to the 3 -tuple $\mathcal{A}, \epsilon, e_{n+1}$, and define $\epsilon^{\prime}=\gamma / 7 n^{2}(1+\mu)^{2}$. We may assume that $\gamma<1$. Define $\delta(\epsilon, J)>0$ by $\delta(\epsilon, J)=\min \left\{\epsilon^{\prime}, \delta\left(\epsilon^{\prime}, I\right)\right\}$, where 
$I=\left\{e_{i} \mid 1 \leq i \leq n\right\}$ and $\delta\left(\epsilon^{\prime}, I\right)>0$ is the positive number given by the induction hypothesis. Let $\mathcal{B}$ be a unital $p$-adic Banach subalgebra of $\mathcal{A}$ such that each member of $\mathcal{B}$ is $\mathcal{A}$-analytic, and let $\left\{a_{i} \mid 1 \leq i \leq n+1\right\}$ be a family of elements in $\mathcal{B}$ such that $\left\|e_{i}-a_{i}\right\| \leq \delta(\epsilon, J)$ for $1 \leq i \leq n+1$. By the induction hypothesis, we can find an orthogonal family $\left\{f_{i} \mid 1 \leq i \leq n\right\}$ of idempotents in $\mathcal{B}$ such that $\left\|f_{i}-e_{i}\right\|<\epsilon^{\prime}$ for $1 \leq i \leq n$. Define $f=\sum_{i=1}^{n} f_{i}$, then we have

$$
\begin{aligned}
& \left\|e_{n+1}-(1-f) a_{n+1}(1-f)\right\| \\
= & \left\|e_{n+1}-(1-f) e_{n+1}(1-f)+(1-f)\left(e_{n+1}-a_{n+1}\right)(1-f)\right\| \\
\leq & \left\|e_{n+1}-(1-f) e_{n+1}(1-f)\right\|+\left\|(1-f)\left(e_{n+1}-a_{n+1}\right)(1-f)\right\| \\
\leq & (1+\|f\|) \cdot\left\|f e_{n+1}\right\|+\left\|e_{n+1} f\right\|+(1+\|f\|)^{2}\left\|e_{n+1}-a_{n+1}\right\| \\
< & 7 n^{2}(1+\mu)^{2} \epsilon^{\prime} \\
< & \gamma .
\end{aligned}
$$

Let $\mathcal{C}$ be the unital commutative $p$-adic Banach subalgebra of $\mathcal{B}$ generated by the family

$$
\left\{1,(1-f) a_{n+1}(1-f)\right\} \cup\left\{f_{i} \mid 1 \leq i \leq n\right\} .
$$

Then each member of $\mathcal{C}$ is $\mathcal{A}$-analytic. By our choice of $\gamma$, Theorem $3.17 \mathrm{im}$ plies that there exists an idempotent $f_{n+1} \in \mathcal{C}$ such that $\left\|f_{n+1}-e_{n+1}\right\|<\epsilon$. Moreover, for $1 \leq i \leq n$, we have

$$
\begin{aligned}
\left\|f_{n+1} f_{i}\right\| & \leq\left(\left\|f_{i}-e_{i}\right\|+\left\|e_{i}\right\|\right) \cdot\left\|f_{n+1}-e_{n+1}\right\|+\left\|e_{n+1}\right\| \cdot\left\|f_{i}-e_{i}\right\| \\
& <(\delta(\epsilon, J)+\mu) \delta(\epsilon, J)+\mu \delta(\epsilon, J) \\
& <7 n^{2}(1+\mu)^{2} \epsilon^{\prime} \\
& <\gamma<1 .
\end{aligned}
$$

Because $\mathcal{C}$ is commutative, for $1 \leq i \leq n, f_{n+1} f_{i}$ is an idempotent, hence we must have $f_{n+1} f_{i}=0$. Thus $\left\{f_{i} \mid 1 \leq i \leq n+1\right\}$ is an orthogonal family of idempotents in $\mathcal{B}$ such that $\left\|e_{i}-f_{i}\right\|<\epsilon$ for $1 \leq i \leq n+1$. This completes the proof of the lemma.

\section{On Classifying $p$-Adic Triangular UHF Algebras}

We are now ready to state the the main result of the the present paper, namely, Theorem 4.2. We will use the following terminology: Let $\mathcal{A}$ be a unital $p$ adic Banach algebra over $\Omega_{p}$ and let $\mathcal{B}$ be a $p$-adic Banach subalgebra of $\mathcal{A}$ 
which contains the identity of $\mathcal{A}$. Then $\mathcal{B}$ is said to be a full matrix algebra if there exists a positive integer $n$ such that $\mathcal{B}$ is isomorphic to $M_{n}\left(\Omega_{p}\right)$ as an $\Omega_{p}$-algebra. Throughout the remainder of this paper, all $p$-adic Banach algebras will be unital.

Definition 4.1. Let $\mathcal{B}$ be a $p$-adic Banach algebra over $\Omega_{p}$, and let $\mathcal{T} \subseteq \mathcal{B}$ be a $p$-adic TUHF Banach algebra given by

$$
\mathcal{T}=\overline{\bigcup \mathcal{T}_{n}}, \quad \mathcal{T}_{n}=\left\langle f_{i j}^{(n)} \mid 1 \leq i \leq j \leq q_{n}\right\rangle, \quad\left(\mathcal{T}_{n}\right) \text { increasing. }
$$

Let $\mathcal{G}$ be a family of finite-dimensional unital $p$-adic Banach subalgebras of $\mathcal{B}$ such that each member of $\mathcal{G}$ contains the identity of $\mathcal{B}$ and is a full matrix algebra. We say that the family $\mathcal{G}$ satisfies the local dimensionality condition with respect to $\mathcal{T}$ if the following conditions hold.

(i) For each $n$, there exists $\mathcal{N} \in \mathcal{G}$ such that $\mathcal{T}_{n} \subseteq \mathcal{N}$ and the triangular system of matrix units $\left\{f_{i j}^{(n)} \mid 1 \leq i \leq j \leq q_{n}\right\}$ can be extended to a system of matrix units $\left\{f_{i j}^{(n)} \mid 1 \leq i, j \leq q_{n}\right\}$ in $\mathcal{N}$.

(ii) Let $m$ be a positive integer such that $\mathcal{T}_{m} \subseteq \mathcal{M}$, where $\mathcal{M} \in \mathcal{G}$. Then for any $n \geq m$, there exists $\mathcal{N} \in \mathcal{G}$ such that $\mathcal{M}, \mathcal{T}_{n} \subseteq \mathcal{N}$.

Theorem 4.2. Let $\mathcal{B}$ be a unital $p$-adic Banach algebra over $\Omega_{p}$, and let $\mathcal{T} \subseteq \mathcal{B}$ be a $p$-adic $\mathrm{TUHF}$ Banach subalgebra of $\mathcal{B}$ given by

$$
\mathcal{T}=\overline{\bigcup \mathcal{T}_{n}}, \quad \mathcal{T}_{n}=\left\langle f_{i j}^{(n)} \mid 1 \leq i \leq j \leq q_{n}\right\rangle, \quad\left(\mathcal{T}_{n}\right) \text { increasing. }
$$

Let $\mathcal{G}$ be a family of finite-dimensional p-adic Banach subalgebras of $\mathcal{B}$ such that each member of $\mathcal{G}$ contains the identity of $\mathcal{B}$ and is a full matrix algebra. Assume that $\mathcal{G}$ satisfies the local dimensionality condition with respect to $\mathcal{T}$. Let $\mathcal{S}$ be a $p$-adic TUHF Banach algebra given by

$$
\mathcal{S}=\overline{\bigcup \mathcal{S}_{n}}, \quad \mathcal{S}_{n}=\left\langle e_{i j}^{(n)} \mid 1 \leq i \leq j \leq p_{n}\right\rangle, \quad\left(\mathcal{S}_{n}\right) \text { increasing. }
$$

Suppose that there exists an algebraic isomorphism $\Phi: \mathcal{S} \rightarrow \mathcal{T}$ such that $\overline{\bigcup \Phi\left(\mathcal{S}_{n}\right)}=\mathcal{T}$. Then $N\left[\left(q_{n}\right)\right] \leq N\left[\left(p_{n}\right)\right]$. Moreover, if there exists a unital $p$-adic Banach algebra $\mathcal{A}$ such that $\mathcal{S} \subseteq \mathcal{A}$ and a family $\mathcal{F}$ of finite-dimensional $p$-adic Banach subalgebras of $\mathcal{A}$ such that each member of $\mathcal{F}$ is a full matrix algebra containing the identity of $\mathcal{A}$ and $\mathcal{F}$ satisfies the local dimensionality condition with respect to $\mathcal{S}$, then there exists an $\Omega_{p}$-algebra isomorphism $\Psi: \mathcal{T} \rightarrow \mathcal{S}$ such that $\overline{\bigcup \Psi\left(\mathcal{T}_{n}\right)}=\mathcal{S}$, only if $N\left[\left(p_{n}\right)\right]=N\left[\left(q_{n}\right)\right]$.

Using the $p$-adic Banach-Steinhaus theorem (see [15], Theorem 3.12), p-adic Banach algebra inductive limits are constructed in the same way that they are 
for complex Banach algebras ([Bl], Section 3.3). The next lemma provides a means of constructing a large class of $p$-adic triangular UHF algebras such that Theorem 4.2 applies to each member of this class. The proof of Lemma 4.3 is routine.

Lemma 4.3. Let $\left(p_{n}\right)$ be a sequence of positive integers, and let $\left(\|\cdot\|_{n}\right)$ be a sequence of $p$-adic Banach algebra norms on $M_{p_{n}}\left(\Omega_{p}\right)$. Let $\left\{\Phi_{n m} \mid m \leq n\right\}$ be a family of continuous, unital monomorphisms $\Phi_{n m}:\left(M_{p_{m}}\left(\Omega_{p}\right) ;\|\cdot\|_{m}\right) \rightarrow$ $\left(M_{p_{n}}\left(\Omega_{p}\right) ;\|\cdot\|_{n}\right)$, such that the following conditions hold.

(i) $\Phi_{n m}\left(T_{p_{m}}\left(\Omega_{p}\right)\right) \subseteq T_{p_{n}}\left(\Omega_{p}\right)$, for $m \leq n$;

(ii) $\Phi_{n p} \Phi_{p m}=\Phi_{n m}$, for $m \leq p \leq n$;

(iii) $\limsup \left\|\Phi_{n m}\right\|<+\infty$ for all $m$. $n \geq m$

Let $\mathcal{B}$ be the $p$-adic Banach algebra inductive limit $\underset{\longrightarrow}{\lim }\left(M_{p_{n}}\left(\Omega_{p}\right) ;\|\cdot\|_{n} ; \Phi_{n m}\right)$, and write $\mathcal{B}=\overline{\cup \mathcal{N}_{n}}$, where $\mathcal{N}_{n}$ is the canonical image of $M_{p_{n}}\left(\Omega_{p}\right)$ in $\mathcal{B}$. Define $\mathcal{G}$ to be the family $\left\{\mathcal{N}_{n} \mid n \geq 1\right\}$. Finally, let $\mathcal{T}=\overline{U \mathcal{T}_{n}}$, where $\mathcal{T}_{n}$ is the canonical image of $T_{p_{n}}\left(\Omega_{p}\right)$ in $\mathcal{B}$. Then $\mathcal{T}$ is a $p$-adic TUHF Banach algebra over $\Omega_{p}$ such that the triple $\mathcal{B}, \mathcal{T}, \mathcal{G}$ satisfies conditions (i) and (ii) of Theorem 4.2 .

We are now ready to present a purely $p$-adic Banach-algebraic formulation of the main result in [2]. In for any positive integer $n$, set $\Omega_{p}^{n}=\Omega_{p}(\{1,2, \ldots, n\})$. Then by Definition 3.2, $\Omega_{p}^{n}$ is a $p$-adic Banach algebra over $\Omega_{p}$ under the norm

$$
\left\|\left(x_{1}, \ldots, x_{n}\right)\right\|_{p}=\max _{1 \leq i \leq n}\left|x_{i}\right|_{p} .
$$

We identify the $\Omega_{p}$-algebra $M_{n}\left(\Omega_{p}\right)$ of $n \times n$ matrices over $\Omega_{p}$ with $B\left(\Omega_{p}^{n}\right)$. Then the $\Omega_{p}$-algebra $T_{n}\left(\Omega_{p}\right)$ of $n \times n$ upper triangular matrices over $\Omega_{p}$ is identified with a $p$-adic Banach subalgebra of $M_{n}\left(\Omega_{p}\right)$.

Proposition 4.4. For each pair of positive integers $m, n$ such that $m \mid n$, let there correspond a unital monomorphism $\Theta_{m n}: M_{m}\left(\Omega_{p}\right) \rightarrow M_{n}\left(\Omega_{p}\right)$ such that the following conditions are satisfied.

(i) $\Theta_{m n}\left(T_{m}\left(\Omega_{p}\right)\right) \subseteq T_{n}\left(\Omega_{p}\right)$;

(ii) if $m|r| n$, then $\Theta_{m r} \Theta_{r, n}=\Theta_{m n}$;

(iii) $\sup \left\{\left\|\Theta_{m n}\right\|_{p},\left\|\Theta_{m n}^{-1}\right\|_{p}: m \mid n\right\}<\infty$.

Let $\left(p_{n}\right)$ and $\left(q_{n}\right)$ be sequences of positive integers such that $p_{m} \mid p_{n}$ and $q_{m} \mid q_{n}$ whenever $m \leq n$. Define $\mathcal{S}$ and $\mathcal{T}$ to be the TUHF Banach algebras

$$
\mathcal{S}=\lim _{\longrightarrow}\left(T_{p_{n}}\left(\Omega_{p}\right) ; \Theta_{p_{n} p_{m}}\right), \quad \mathcal{T}=\lim _{\longrightarrow}\left(T_{q_{n}}\left(\Omega_{p}\right) ; \Theta_{q_{n} q_{m}}\right) .
$$


For each $n$ let $\mathcal{S}_{n}=\Phi_{n}\left(T_{p_{n}}\left(\Omega_{p}\right)\right)$ and $\mathcal{T}_{n}=\Psi_{n}\left(T_{q_{n}}\left(\Omega_{p}\right)\right)$, where $\Phi_{n}$ (resp., $\Psi_{n}$ ) is the canonical mapping of $T_{p_{n}}\left(\Omega_{p}\right)$ (resp., $T_{q_{n}}\left(\Omega_{p}\right)$ ) into $\mathcal{S}$ (resp., $\mathcal{T}$ ). Then there exist $\Omega_{p}$-algebra isomorphisms $\Phi: \mathcal{S} \rightarrow \mathcal{T}$ and $\Psi: \mathcal{T} \rightarrow \mathcal{S}$ such that $\overline{\bigcup \Phi\left(\mathcal{S}_{n}\right)}=\mathcal{T}$ and $\overline{\bigcup \Psi\left(\mathcal{T}_{n}\right)}=\mathcal{S}$, if, and only if $N\left[\left(p_{n}\right)\right]=N\left[\left(q_{n}\right)\right]$.

Proof. The proof of Proposition 4.4 is a simple adaptation of the proof of Proposition 3.5 in [3].

Let $p, q$ be positive integers such that $q \mid p$; define $\rho_{p q}, \sigma_{p q}: M_{q}\left(\Omega_{p}\right) \rightarrow M_{p}\left(\Omega_{p}\right)$ by $\rho_{p q}(x)=x \otimes 1_{d}, \sigma_{p q}(x)=1_{d} \otimes x, x \in M_{q}\left(\Omega_{p}\right)$ and $d=p / q$. Then Proposition 4.4 applied to the mappings $\sigma_{p q}$ yields the exact $p$-adic counterpart of the main result in [2]. On the other hand, Proposition 4.4 applied to the mappings $\rho_{p q}$ yields a classification of $p$-adic TUHF Banach algebras of the form $\mathcal{T}=\underset{\longrightarrow}{\lim }\left(T_{p_{n}}\left(\Omega_{p}\right) ; \rho_{p_{n} q_{m}}\right)$, where $\left(p_{n}\right)$ is a sequence of positive integers such that $p_{m} \mid p_{n}$ whenever $m \leq n$; algebras of this form are also classified in [13] and in $[11],[12]$.

\section{References}

[1] D.J. Alkers, G.L. Alexnaderson, C. Reid, More Mathematical People, Harcourt Brace Jovanovich, 1990.

[2] R. Baker, Triangular UHF algebras, J. Funct. Anal., 91, No. 1 (1990), $182-212$.

[3] R.L. Baker, On certain Banach limits of triangular matrix algebras, Houston J. Math., 23, No. 1 (1997), 127-141.

[4] R.L. Baker, A certain p-adic spectral theorem, IJPAM, 75, No. 1 (2012), 19-68.

[5] R.L. Baker, On the classification of $p$-adic UHF Banach algebras, Preprint.

[6] B. Blackadar, K-Theory for Operator Algebras, Springer-Verlag, New York, 1986.

[7] N. Dunford, J.T. Schwartz, Linear Operators Part I, Interscience Publishers, Inc., New York, 1963.

[8] J. Glimm, On a certain class of operator algebras, Trans. Amer. Math. Soc., 95 (1960), 318-340. 
[9] N. Koblitz, p-adic Analysis: A Short Course on Recent Work, London Mathematical Society Lecture Note Series, 46, Cambridge University Press, New York, 1980.

[10] L. Narici, E. Beckenstein, G. Bachman, Functional Analysis and Valuation Theory, Marcel Dekker, Inc., New York, 1971.

[11] S.C. Power, Classifications of tensor products of triangular matrix algebras, Proc. London Math. Soc., 61, No. 3 (1990), 571-614.

[12] S.C. Power, The classification of triangular subalgebras of $C^{*}$-algebras, Bull London Math. Soc., 22 (1990), 269-272.

[13] J.R. Peters, Y.T. Poon, B.H. Wagner, Triangular AF algebras, J. Operator Theory, 23 (1990), 81-114.

[14] A.M. Robert, A Course in p-Adic Analysis, Springer-Verlag, New York, Inc., 2000.

[15] A.C.M. van Rooij, Non-Archimedean Functional Analysis, Marcel Dekker, Inc., New York, 1978.

[16] L.G. Shnirel'man, O funckcijah v normirovannyh algegraičeski zamknutyh telah (On functions in normed algebraically closed division rings), Izvestija AN SSSR, 2 (1938), 487-498. 
